\title{
It's time for a national immunization strategy
}

\author{
Joanne Embree MD FRCPC
}

$\mathrm{C}^{\mathrm{a}}$ anada needs to develop and implement a national immunization strategy to provide optimal protection for vaccine-preventable diseases for children, adolescents and adults. Although the federal government has a mandate to ensure that access to health care is equitable across the country, it is the mandate of provincial governments to actually provide health care services. Thus, while the federal government makes recommendations for the optimal use of various vaccines through the publications of the National Advisory Committee on Immunization, the provincial governments must develop and fund their individual immunization programs. With a national immunization strategy that is endorsed by the federal and provincial governments, the following could occur: harmonization of childhood immunization schedules across the country; efficient introduction of new vaccines; the ability to enhance the monitoring of vaccine use and adverse events; and improvements in the ability to access readily vaccine products in the most cost effective manner. A national immunization strategy would also provide opportunities for vaccine and immunization research, and for improved education of health care providers and the general public.

The use of immunization to prevent infectious diseases is the most cost effective medical intervention available to public health. In Canada, vaccines against polio, tetanus, diphtheria, pertussis, measles, mumps, rubella, Haemophilus influenzae type $\mathrm{b}$ and hepatitis $\mathrm{B}$ infection are routinely offered to all children; however, vaccine schedules vary from province to province. In addition, many Canadian children and adults receive vaccines to prevent other diseases, such as varicella, influenza, tuberculosis, hepatitis A and invasive Streptococcus pneumoniae infections. Recently, vaccine research has resulted in the development of new vaccines with improved protection against meningococcal and pneumococcal illnesses for young children, and one vaccine against pertussis with an improved safety profile.
Unfortunately, all of these successes have resulted in problems that were unforeseen a decade ago. With the use of universal vaccination, illnesses that plagued previous generations have been virtually eliminated from the minds of most of the general public. This substantially lower risk of disease has resulted in the need to ensure maximum vaccine safety to sustain the current high rates of vaccine coverage. Otherwise, doubts about vaccine safety may result in the reemergence of illness due to these vaccine-preventable infections. The cost of new vaccine development has escalated due to technological complexity, and the need for extensive efficacy and safety trials before licensure. Increased demand for specific vaccines and a lack of competition in the market place may also play a significant role in the cost of vaccines. These factors have resulted in delays and inequities in the implementation of some universal vaccine programs as the various provinces individually struggle with prioritizing the universal introduction of costly vaccines into their health budgets. As a result, factors such as residence or family income can become the determinants of vaccine availability, resulting in inequitable access to new vaccines.

What should be included in a national immunization strategy?

\section{GOALS OF A NATIONAL IMMUNIZATION STRATEGY} Immunization registry: One of the first initiatives toward developing a national immunization strategy should be to create a reliable, up-to-date registry system for immunizations. Optimal implementation of other aspects of a national strategy depends on the presence of such a registry. The province of Manitoba has an example of such a registry in the Manitoba Immunization Monitoring System. Initially, this system only involved the registration of routine childhood vaccines but was recently expanded to include vaccines provided to adults. This system could be easily copied or modified for use by other provinces. To use these immuniza- 
tion registries optimally, linkages should be established among the different provincial registry systems. This would enable vaccine providers to determine which vaccines their patients had actually received and when, regardless of where they had been immunized. These registries would then provide a backup for those situations in which personal immunization records have been lost and are needed for purposes such as school or university entry. Properly designed registries would also be used to track regional and national immunization levels for individual vaccines while maintaining each individual's privacy rights. These registries would also provide powerful tools to monitor the effects of immunization on disease incidence. As well, the registries are essential to the development of a more comprehensive system of vaccine safety monitoring. Such a system is not possible without accurate knowledge of the vaccine type, lot number and date of vaccine administration to both affected and unaffected individuals. Bar coding of vaccines would make this process both easier and less prone to error.

Vaccine safety monitoring: The second goal of a national immunization strategy should be the further development of an integrated, comprehensive program to monitor, evaluate and investigate vaccine safety issues following licensure. Unfortunately, the claims made by some individuals have undermined the public's confidence in some immunization programs. Often the allegations are not based on testable hypotheses, and the most vocal individuals are often not swayed by scientific explanations. Be that as it may, it is important to ensure that the vaccines administered in the immunization programs are as safe as possible. Presently, a number of systems monitor vaccine adverse events in Canada. Vaccine manufacturers accept reports of adverse events and forward them to Health Canada. The Vaccine Associated Adverse Events Surveillance System is a passive reporting system through which Canadian physicians and other vaccine providers report suspected vaccine adverse events to local and provincial authorities who, in turn, report the events to the Division of Immunization of Health Canada's Population and Public Health Branch. This system is dependent on voluntary reporting, which may result in substantial under-reporting. The Advisory Committee on Causality Assessment assesses all serious individual reported adverse events to determine the likelihood that they were caused by a vaccine. This is often a difficult task if the necessary information needed to make the assessment is not available. There is also an active surveillance program for vaccine adverse events that involves 12 sentinel paediatric hospitals and health centres - the Immunization Monitoring Program ACTive. This program is coordinated by the Canadian Paediatric Society and funded by the Division of Immunization of Health Canada's Population and Public Health Branch (one centre is funded by the province of Alberta). In this program, nurse monitors assess all admitted patients for vaccine adverse events, as well as vaccinepreventable illnesses and vaccine failures. Thus, the program has the advantage of active surveillance with complete data reporting but is restricted to hospitalized children. The Canadian Paediatric Surveillance Network, another collaborative initiative between the Canadian Paediatric Society and Health Canada, has the capacity to monitor for the incidence of known rare adverse events, even though it has not yet been used for this purpose.

There is a need to monitor vaccine safety systematically with a system that allows for the timely notification of health care providers when a problem is first suspected or identified. As well, capacity building in the research community is necessary so that common or serious true vaccine-associated adverse events can be systematically studied to determine their causes and the means to prevent or modify them. A national strategy on vaccine safety must also include the issue of how to effectively communicate with the public as a priority. Effective communication is important to the minority of parents who question whether their children should be vaccinated, as well as for the general public.

Improved vaccine procurement: Ensuring optimal vaccine procurement processes should be another component of an effective national immunization strategy. Presently, concerns are related to bargaining for the best available vaccine price, and to the accessibility of both new and established vaccines. Vaccine prices may vary considerably from province to province, depending on the asking price at the time when contract negotiations took place and the number of doses required by a province; larger orders over a longer contract period get a more favourable price, thus, unfairly penalizing smaller provinces. In some situations, sufficient doses of vaccines may just not be available. The above issues could be resolved with a national strategy on vaccine procurement, which has the goal of ensuring the supply of both new and established vaccines to publicly funded immunization programs.

Harmonization of immunization schedules: Harmonization of the provincial childhood immunization schedules should be an additional goal of a national immunization strategy. The advantages of this approach are obvious, and include equal access to vaccines across the country and limiting the number of missed vaccinations when children move from province to province. One of the most important barriers to harmonization and equitable access to childhood vaccines is the absence of a national process to move from the recommendations published in a National Advisory Committee on Immunization statement to a nationally agreed position on vaccine use. Having such a process to evaluate new vaccines from a program perspective and to prioritize the introduction of new products is the most important step in establishing a uniform approach to immunization across the country. The negotiations to establish such a program will be complex because they will involve all of the provinces and territories, as well as the federal government. However, in the long term, the maximum harmonization of the immunization schedules will be well worth the effort involved.

Research initiatives: The establishment of the Canadian Institutes of Health Research provided the impetus for researchers with an interest in vaccine issues to develop the 
Canadian Association for Immunization Research and Evaluation. The commitment of sufficient funds to enable this group to conduct appropriate studies before and following the introduction of new or improved vaccines should be research priorities. Studies on vaccine safety, vaccine usage, correlates of protection, optimal immunization schedules, cost benefit analyses and disease epidemiology are research priorities. The strategy should also have an international research and development component, because all Canadians have a responsibility to facilitate the development and implementation of vaccines for the developing world.

Education: Another aspect of a national immunization strategy is the establishment of an educational strategy for nursing and medical students that emphasizes the rationale for various immunization programs, the risks and benefits of individual vaccines, and the concept of causality assessment when evaluating adverse events temporally associated with vaccine administration. As well, there is a need for health care providers to learn how to communicate effectively with people who are reluctant to be immunized in both private and public circumstances. Finally, strengthening the capacity for public education in general and appropriate awareness campaigns concerning emerging immunization issues are also important components of a national immunization educational strategy.

\section{CONCLUSION}

It should be the right of every child in Canada to have the opportunity to be optimally protected against vaccinepreventable diseases. A well thought out and comprehensive national immunization strategy is the best option for Canada to be able to ensure this right.

\section{INFECTIOUS DISEASES AND IMMUNIZATION COMMITTEE}

Members: Drs Upton Allen, The Hospital for Sick Children, Toronto, Ontario; H Dele Davies, Division of Infectious Diseases, Alberta Children's Hospital, Calgary, Alberta; Joanne Embree, The University of Manitoba, Winnipeg, Manitoba (Chair); Joanne Langley, Department of Pediatrics, IWK Health Centre, Halifax, Nova Scotia; Mireille Lemay, Department of Infectious Diseases, Sainte-Justine Hospital, Montréal, Québec; Gary Pekeles, The Montreal Children's Hospital, Montreal, Quebec (director responsible)

Consultants: Drs Noni MacDonald, Faculty of Medicine, Dalhousie University, Halifax, Nova Scotia; Victor Marchessault, Cumberland, Ontario Liaisons: Drs Scott Halperin, Department of Pediatrics, IWK Health Centre, Halifax, Nova Scotia (IMPACT); Susan King, Division of Infectious Diseases, The Hospital for Sick Children, Toronto, Ontario (Canadian Paediatric AIDS Research Group); Monique Landry, Direction de la santé publique de Laval, Laval, Québec (Public Health); Larry Pickering, Centre for Pediatric Research, Norfolk, Virginia (American Academy of Pediatrics); John Waters, Alberta Health, Edmonton, Alberta (Epidemiology)

Principal author: Dr Joanne Embree, The University of Manitoba, Winnipeg, Manitoba (chair)

This article also appears in Paediatr Child Health 2001;6(6):316-318. 


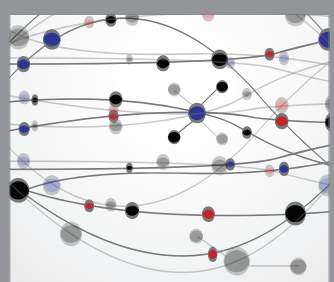

The Scientific World Journal
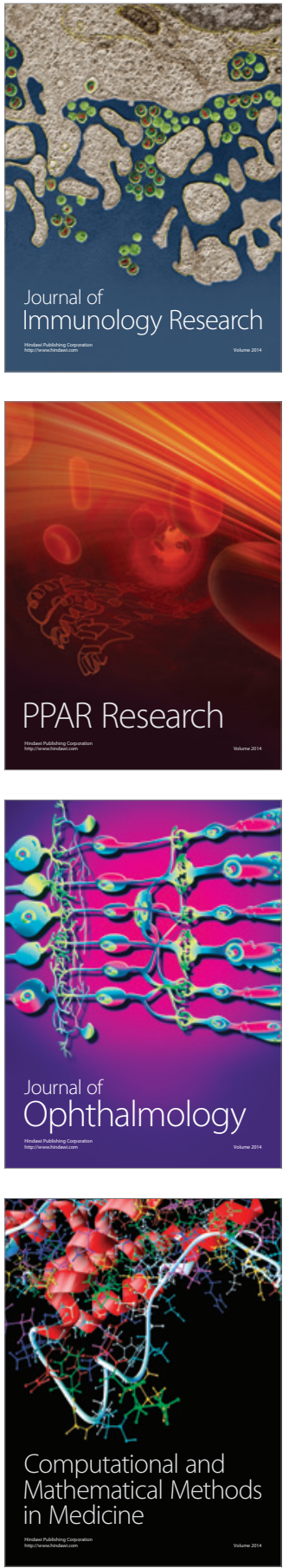

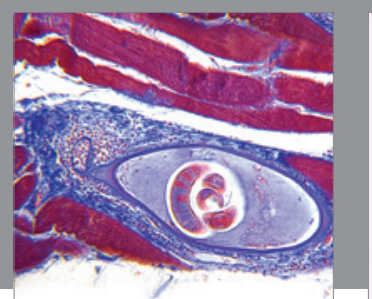

Gastroenterology Research and Practice

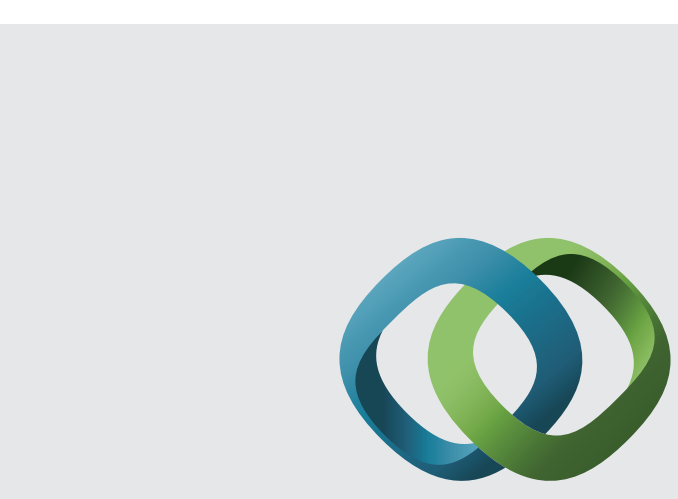

\section{Hindawi}

Submit your manuscripts at

http://www.hindawi.com
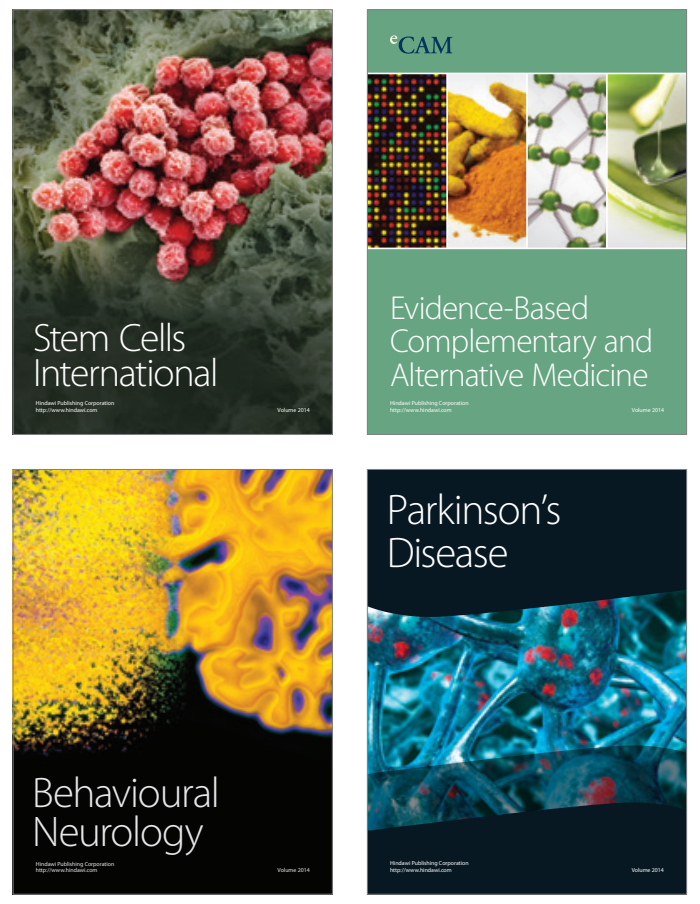
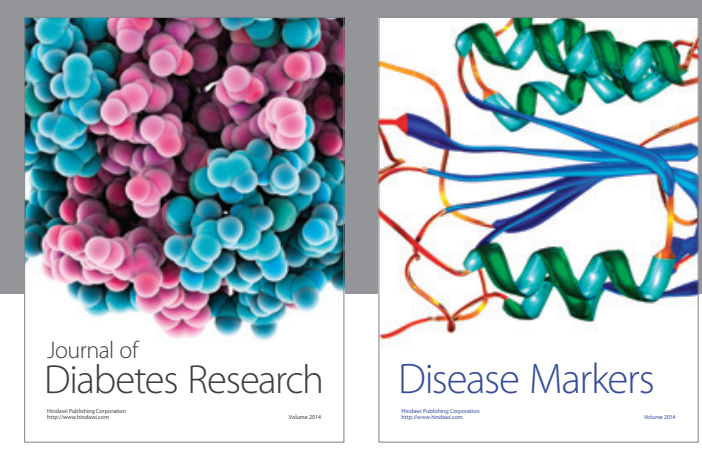

Disease Markers
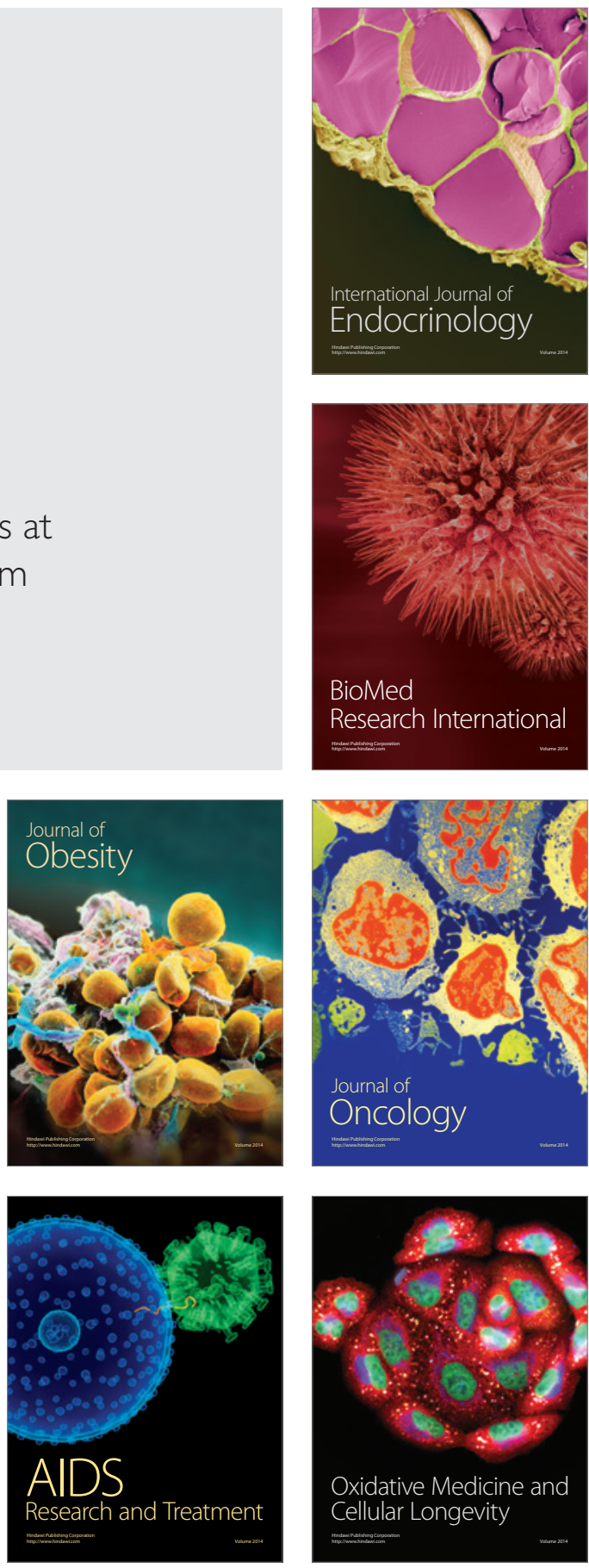